*Correspondencia:

dra.rodriguezv@yahoo.com

Teléfono [593] 022990800

Conflicto de intereses: Los autores declaran no tener conflictos de intereses.

Fondos: Ver la página 178

Recibido: 15 Mayo 2019

Aceptado: 21 Julio 2019

Publicado: 30 Diciembre 2019

Membrete bibliográfico:

Rodríguez D, Frías-Toral E, Santana $S$. Requerimientos ajustados a la Desnutrición del Paciente Oncológico. Rev. Oncol. Ecu 2019;29(3):168-180.

ISSN: 2661-6653

DOI: https://doi.org/10.33821/271

Copyright Rodríguez D, et al Este artículo es distribuido bajo los términos de Creative

Commons Attribution License, el cual permite el uso y

redistribución citando la fuente y al autor original.

\section{Requerimientos ajustados a la Desnutrición del Paciente Oncológico.} \section{Requirements adjusted to the Malnutrition of the Cancer Patient.}

\author{
Dolores Rodríguez Veintimilla1*iD), Evelyn Frías-Toral1id, Sergio \\ Santana Porbén2
}

1. Solca-Guayaquil.

2. Hospital Pediátrico Docente Juan Manuel Marquez de La Habana-Cuba.

\section{Resumen}

Desnutrición y cáncer son 2 términos íntimamente relacionados y resulta importante entender cómo interactúan entre sí ya que pueden afectar negativamente al pronóstico del paciente. Muchas veces por desajustes en el metabolismo del paciente, o por causas relacionadas al tumor o a los mismos tratamientos oncológicos el paciente empeora su estado nutricional. Esto implica aparición de complicaciones, retraso en administración de esquemas terapéuticos. Este artículo explicará las causas de desnutrición en el paciente con cáncer y los requerimientos de energía y nutrientes específicos para esta población.

Palabras Claves: DESNUTRICIÓN, NEOPLASIAS, CONSUMOS DE ENERGÍA, NUTRIENTES.

DOI: $10.33821 / 450$

\section{Abstract}

Malnutrition and cancer are two intimately related terms, and it is essential to understand how they interact with each other since they can negatively affect the patient's prognosis. Imbalances in patient's metabolism causes related to the tumor or the same oncological treatments, are some of the main causes for patients worsening their nutritional status. As a result of these the prevalence of complications increases and the delay in the administration of therapeutic schemes. This article will explain the causes of malnutrition in cancer patients and the specific energy and nutrient requirements for this population.

Keywords: MALNUTRITION, NEOPLASMS, ENERGY CONSUMPTION, NUTRIENTS.

DOI: $10.33821 / 450$ 


\section{Introducción}

La prevalencia de desnutrición en el paciente oncológico es un problema común; se estima que la incidencia de malnutrición oscila entre 15 y $40 \%$ en el momento del diagnóstico del cáncer, y aumenta hasta $80 \%$ en los casos de enfermedad avanzada [1]. Las consecuencias de la desnutrición implicarán la aparición de un mayor número de complicaciones en el período posterior a la operación, estancias hospitalarias más largas, mayor costo económico y una mortalidad más elevada [2].

\section{Causas de desnutrición en pacientes con Cáncer}

Existen tres segmentos que deben tratarse al describir las causas de desnutrición en el paciente neoplásico [3], estos mecanismos están representados en la Figura 1 y se irán describiendo a los largo de la presente revisión. Se pueden concretar en:

1) Los mecanismos básicos de desnutrición en el cáncer, son:

- Escaso aporte de energía y nutrientes.

- Alteraciones de la digestión y/o absorción de nutrientes.

- Aumento de las necesidades.

- Alteraciones en el Metabolismo de los nutrientes.

\section{Causas de desnutrición relacionadas al Tumor}

Las causas de desnutrición relacionadas con el tumor, con el paciente o con los tratamientos, donde se refleja la gran complejidad de la desnutrición en el cáncer y cómo en muchas ocasiones casi todas las causas pueden estar presentes en un mismo paciente.

\section{Alteraciones del Aparato Digestivo}

La existencia de un cáncer en cualquier punto del aparato digestivo puede inducir alteraciones mecánicas o funcionales que alteran de forma clara la alimentación del paciente. En los tramos más proximales los tumores de cabeza y cuello son uno de los cánceres que inducen con mayor frecuencia desnutrición, tanto por alteración en la masticación, salivación o deglución como por el dolor que con gran frecuencia se asocia [4].

\section{Alteraciones metabólicas.}

El metabolismo del paciente canceroso puede experimentar importantes alteraciones como consecuencia de la presencia del tumor. 
Figura 1.- Génesis Multifactorial de la Desnutrición y Caquexia causada por el tumor.

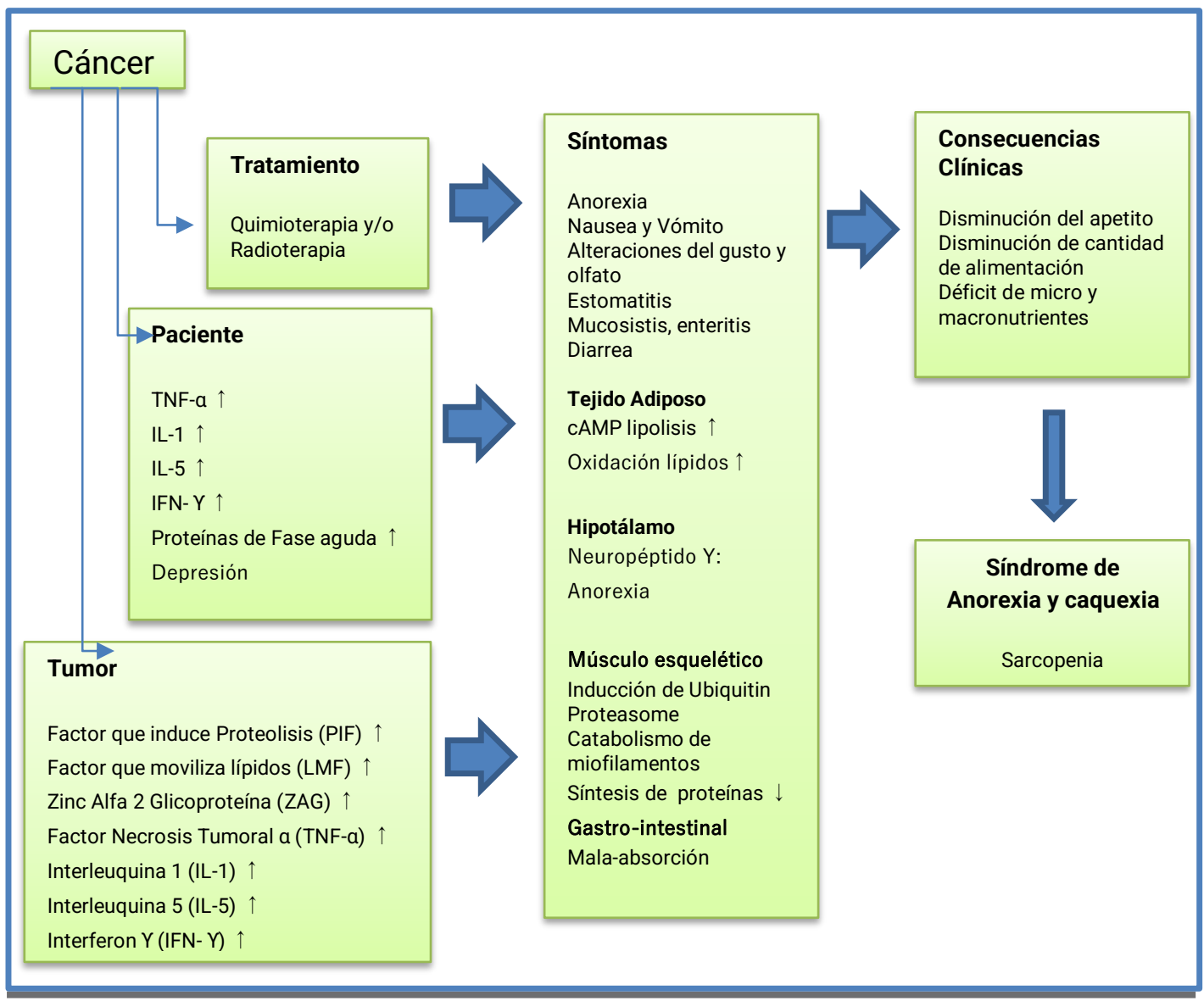

A pesar de la malnutrición que acompaña al crecimiento tumoral avanzado, estos cambios son en muchos aspectos diferentes a los que se presentan típicamente en las situaciones de ayuno, siendo más parecidos a los que tienen lugar en respuesta a una situación de inflamación, infección o lesiones traumáticas.

De este modo, una de las principales características de la caquexia cancerosa es el desgaste tisular que sufre el paciente, que afecta particularmente al músculo esquelético y al tejido adiposo, mientras que otros órganos (hígado, bazo, riñón y adrenales) pueden llegar incluso a aumentar su peso transitoriamente.

Las alteraciones metabólicas representan el aspecto más peculiar e importante de la caquexia cancerosa, ya que incluso en ausencia de malnutrición pueden determinar por sí mismas un balance energético y nitrogenado negativo, junto con un grave deterioro del organismo. Por otra parte, si bien la anorexia es un componente casi universal del síndrome caquéctico, ella sola no explica el notable desgaste observado en el paciente, ya que los patrones de pérdida de peso y los cambios en la composición corporal son diferentes de los característicos de la inanición, y sus efectos catabólicos tampoco pueden revertirse mediante la administración de un complemento calórico. 
De este modo, prevalece el punto de vista de que la caquexia cancerosa es principalmente debida a las alteraciones metabólicas producidas por la presencia del tumor. Entre éstas, se observa en el paciente tumoral una incrementada lipólisis, favorecida por una disminución de la actividad de la Lipoproteína Lipasa (LPL) del Tejido Adiposo Blanco (TAB) y que propicia un aumento de los niveles de Triacilgliceroles (TAG) circulantes. Asimismo, se produce un aumento en el recambio proteico tisular y una incrementada utilización hepática del lactato, generado en grandes cantidades por el tumor.

Durante mucho tiempo se pensó que estos desajustes metabólicos eran causados por algún factor segregado por el propio tumor, o bien como resultado de la competencia por los nutrientes entre las células del tumor y las del paciente, pero en la actualidad se sabe que estos factores proceden principalmente de la respuesta del paciente al crecimiento tumoral.

En resumen podemos mencionar cambios metabólicos como Intolerancia a la glucosa, aumento de la gluconeogénesis hepática, activación lipolítica adiposa, pérdida de proteínas musculares, alteraciones hormonales, presencia de respuesta inflamatoria (proteínas de fase aguda y citoquinas)(Figura 2) [5].

Figura 2.- Mediadores Catabólicos en Cáncer

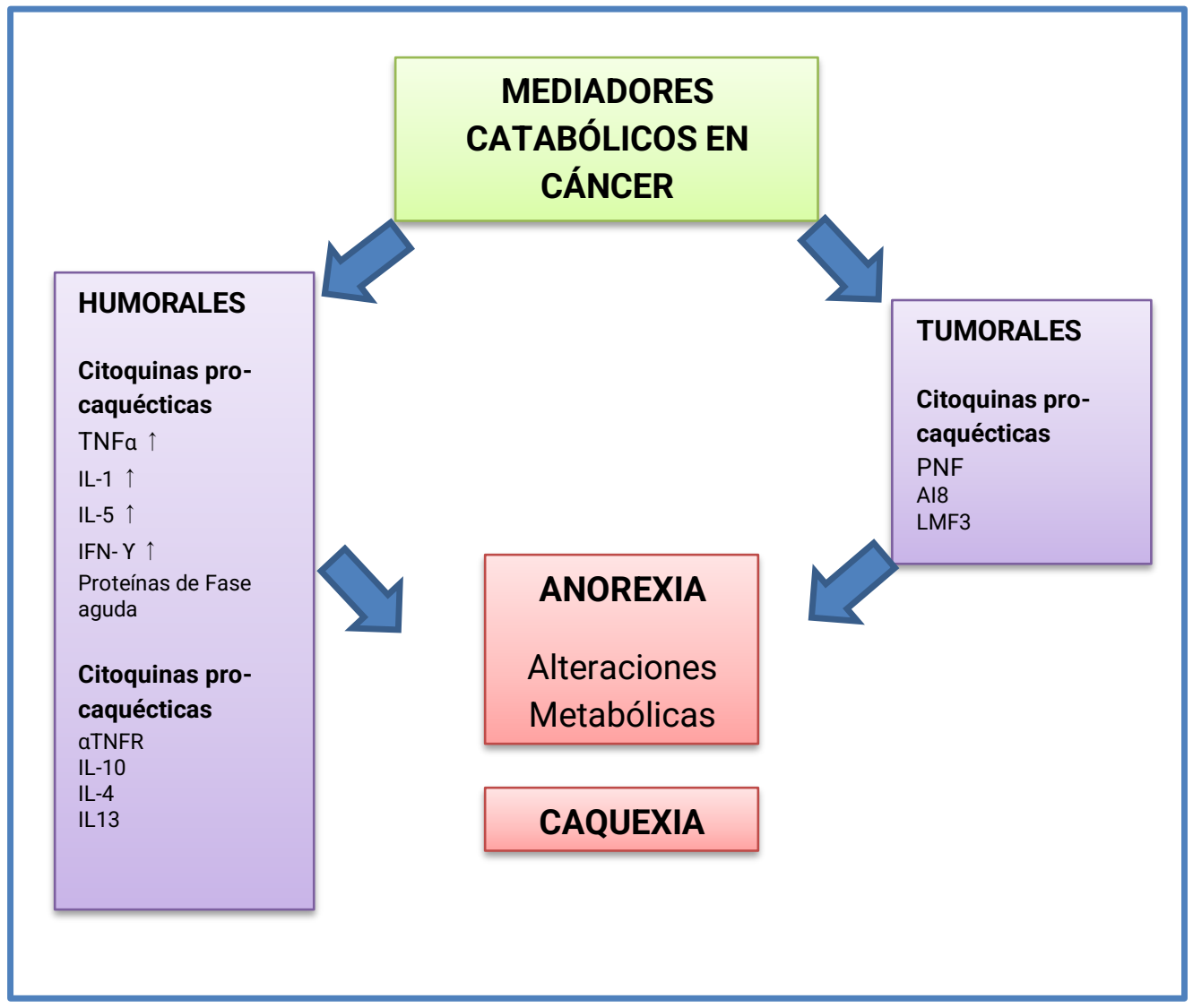




\section{Producción de sustancias caquectizantes.}

El TNF-a fue identificado en 1985 como una proteína sérica presente en animales tratados con endotoxina y que era capaz de inducir necrosis hemorrágica en tumores experimentales. Luego, se determinó que era también la molécula responsable del síndrome de la caquexia asociada a la infección crónica, por lo que también fue bautizada como caquectina [6]. El TNF-a es sintetizado principalmente por los macrófagos en respuesta a distintos estímulos invasivos, como una proteína de $26 \mathrm{kDa}$ ligada a la membrana, que por proteólisis da lugar a una forma madura de $17 \mathrm{kDa}$, si bien su forma biológicamente activa es el trímero $(51 \mathrm{kDa})$. Dicha molécula puede ser reconocida por dos tipos de receptores: de tipo 1 (TNFR1 o p55) y de tipo 2(TNFR2 o p75). El TNF-a es un factor pleiotrópico, con variados efectos sobre el crecimiento celular, angiogénesis, citotoxicidad, inflamación e inmunomodulación.

Aunque las distintas evidencias acumuladas hasta la fecha apuntan a que el TNF-a podría ser el responsable de la mayor parte de los efectos metabólicos observados en situaciones de caquexia, ello no descarta que otros mediadores humorales pudieran también estar implicados. En este sentido, otras citoquinas parecen también tener un papel en este complejo síndrome.

La Interleuquina-6 (IL-6) podría ser una de estas citoquinas, por cuanto la administración de anticuerpos anti-IL- 6 a ratones portadores de un adenocarcinoma de colon revierte los parámetros asociados al estado caquéctico. Sin embargo, otros estudios similares no han podido corroborar estos resultados. Por otra parte, la IL-6 tampoco tiene un efecto sobre la proteólisis muscular in vitro, si bien sí que es capaz de estimular la gluconeogénesis en hepatocitos.

Otra citoquina que podría tener un papel en la caquexia es el interferón- $\gamma$ (IFN- $\gamma)$, por cuanto muestra efectos similares (y sinérgicos) a los del TNF-a. Así, se ha visto que puede inhibir la actividad de LPL en células 3T3L1 y la lipogénesis en adipocitos, y que la administración de anticuerpos monoclonales anti-IFN- $\gamma$ es capaz de revertir notablemente el estado caquéctico en ratones portadores del carcinoma pulmonar de Lewis. Además, la implantación en ratones nude de células $\mathrm{CHO}$ que producen IFN- $\gamma$ de manera constitutiva provoca un fuerte estado caquéctico.

También se ha sugerido un papel significativo para el Factor Inhibidor de la Leucemia (por sus siglas en Inglés LIF), ya que se ha visto que tumores que secretan LIF en ratones pueden provocar una respuesta caquéctica.

Otros candidatos a considerar serían el Factor de Crecimiento Transformante- $\beta$ (por sus siglas en Inglés TGF- $\beta$ ), el Factor Neurotrófico Ciliar (por sus siglas en Inglés CNTF) (producido por la glía, pero también por músculo esquelético) y la Interleuquina-1 (IL-1), la cual tiene fuertes efectos anorexigénicos y pirogénicos, aunque se ha visto que la administración del antagonista del receptor de la IL-1 (IL-1 ra) a ratas caquécticas no revierte la caquexia. (Figura 3) [5]. 
Figura 3.- Citoquinas anticaquécticas y procaquécticas [4].

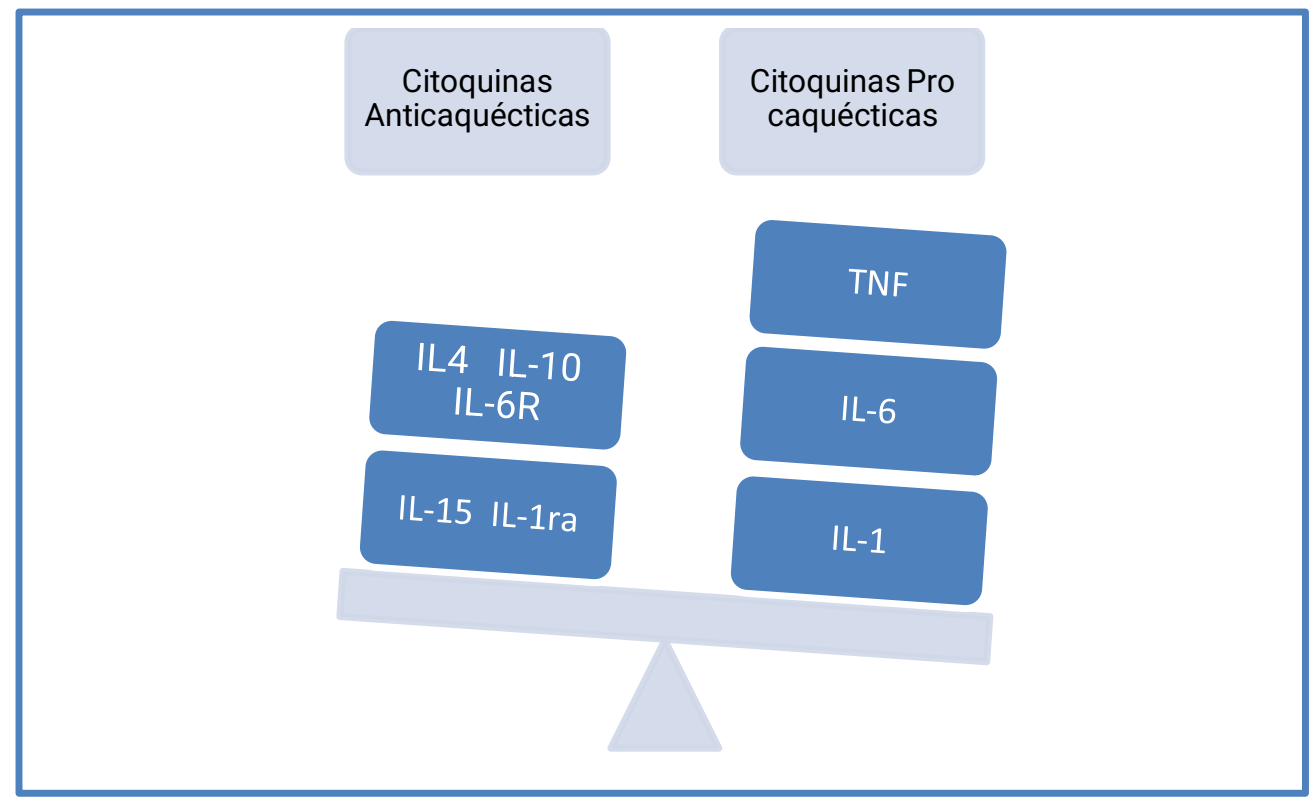

\section{Causas de desnutrición relacionadas con el Paciente}

\section{La Caquexia cancerosa}

Es un complejo síndrome que puede ser la causa directa de casi una cuarta parte de los fallecimientos por cáncer y que se caracteriza por debilidad y pérdida marcada y progresiva de peso corporal, grasa y masa muscular (esquelética y cardíaca); anorexia y saciedad precoz; afectación rápida del estado general (rostro emaciado, piel pálida, rugosa, sin elasticidad, pérdida del vello); alteraciones metabólicas (anemia, edemas, déficit vitaminas, alteración hidroelectrolíticas) y déficit inmunológico (mayor tendencia a infecciones).

Los pacientes mantienen sus gustos alimentarios, pero ingieren menor cantidad de alimento. Se ha estimado que el 20 - 50\% de los pacientes que padecen cáncer experimentan caquexia y alrededor del 65 - $80 \%$ en la fase terminal de la enfermedad. La caquexia no solo aumenta la morbi-mortalidad, sino que disminuye claramente la calidad de vida del paciente oncológico y además aumenta claramente el gasto sanitario [3].

La pérdida de peso se asocia a una disminución de la supervivencia. La caquexia tumoral es responsable directa o indirecta de la muerte de un tercio de los pacientes con cáncer y predice una respuesta pobre al tratamiento oncológico. La valoración nutricional del paciente con cáncer debería comenzar en el momento del diagnóstico y repetirse en cada visita para iniciar la intervención nutricional de forma precoz, antes de que el estado general esté severamente comprometido y las posibilidades de recuperar la situación de normalidad sean pocas. 


\section{Anorexia cancerosa:}

Son las principales causas de desnutrición relacionadas con el paciente. La Anorexia es multifactorial y muy frecuente en el paciente neoplásico. En los cánceres avanzados es casi la norma con cifras que pueden llegar al $70 \%$. El dolor, la medicación, el tratamiento antitumoral y las alteraciones psicológicas pueden aumentar aún más la anorexia, que en muchos casos a pesar de tratar estas causas de anorexia, va a persistir.

Factores psicológicos: Los pacientes cancerosos tienen una gran afectación de la esfera psicológica como consecuencia del propio diagnóstico de cáncer y de la angustia ante los distintos tratamientos a los que va a someterse. Hay que considerar algunos factores psicosociales que pueden provocar una alteración importante en la nutrición como: la depresión, la ansiedad, el miedo son emociones comunes experimentadas por personas con cáncer y pueden contribuir a la anorexia. Así como también cuando viven solos.

\section{Causas de desnutrición producidas por los tratamientos Oncológicos}

\section{Cirugía:}

La cirugía oncológica puede ser curativa o paliativa, y siempre conlleva un estrés metabólico importante sobre todo en el caso de la cirugía curativa, con un aumento de necesidades de energía y nutrientes. Además en muchos pacientes la cirugía se realiza sobre un paciente que ya está malnutrido, lo que potencia de forma clara la aparición de las complicaciones [2].

En cualquier tipo de cirugía son comunes el dolor, la anorexia, la astenia, y algún grado de gastroparesia e íleo paralítico (por la anestesia y por la propia cirugía) que comprometen de alguna manera la ingesta alimentaria normal, y todo ello en un paciente con un aumento de las necesidades energético-proteicas.

Toda cirugía del tracto digestivo es diferente y son más numerosas las alteraciones posquirúrgicas que pueden causar desnutrición. Desde la cirugía de tumores de cabeza y cuello con amplias resecciones o plastias que pueden alterar definitivamente la normal masticación-deglución, hasta todas las resecciones esofágicas, gástricas, pancreáticas, hepáticas y biliares, de intestino delgado, colon y recto (Tabla 1). 
Tabla 1.- Alteraciones de la cirugía oncológica en el tracto digestivo causantes de desnutrición [2]

\begin{tabular}{|l|c|}
\hline \multicolumn{1}{|c|}{ Cirugía } & Alteraciones \\
\hline De cabeza y Cuello & $\begin{array}{r}\text { Alteraciones severas de masticación-deglución. Xerostomía. } \\
\text { Disfagia motora/mecánica. Fístulas }\end{array}$ \\
\hline Esofágica & $\begin{array}{c}\text { Trastornos de la deglución: reflujo, plenitud, saciedad precoz. } \\
\text { Disfagia. Estenosis esofagogástricas. Fístulas: Pleurales. } \\
\text { Mediastinitis. }\end{array}$ \\
\hline $\begin{array}{l}\text { Gástrica } \\
\text { Desecciones del I. }\end{array}$ & $\begin{array}{r}\text { Saciedad Precoz, Síndrome Dumping. Malabsorción B12, Ca, } \\
\text { Fe, Zn. Estenosis. Fístulas. }\end{array}$ \\
\hline $\begin{array}{l}\text { Resecciones } \\
\text { Colon/Recto }\end{array}$ & $\begin{array}{r}\text { Diarrea. Malabsorción. Alteraciones Hidroelectrolíticas. } \\
\text { Intolerancia lactosa. Malabsorción vitaminas liposolubles, } \\
\text { B12, Ca, Fe, Zn. Estenosis. Fístulas. }\end{array}$ \\
\hline Hepática/Biliar & $\begin{array}{r}\text { Diarrea. Malabsorción. Alteraciones Hidroelectrolíticas. } \\
\text { Malabsorción B12, Ca, Mg, Na, K }\end{array}$ \\
\hline Pancreática & $\begin{array}{r}\text { Hiperglucemia. Encefalopatía. Alteraciones } \\
\text { Hidroelectrolíticas. Malabsorción grasa. Déficit Vit } \\
\text { Liposolubles, Ca. }\end{array}$ \\
& $\begin{array}{r}\text { Malabsorción grasa y proteínas. Déficit Vit Liposolubles, Ca, } \\
\text { Mg. Esteatorrea. Hiperglucemia/Diabetes Secundaria. } \\
\text { Fístulas. }\end{array}$ \\
\hline
\end{tabular}

\section{Radioterapia}

El efecto sobre el estado nutricional de la Radioterapia (RT) oncológica depende de la localización del tumor, de la extensión y de la dosis administrada y su fraccionamiento. Además, los efectos secundarios dependerán de los tratamientos que haya recibido el paciente con anterioridad o que se administren concomitantemente con la radioterapia como es el caso de la quimioterapia.

Como una regla general los efectos aparecen en las dos primeras semanas de comenzar la RT, presentan su pico máximo cuando se han administrado los $2 / 3$ de la dosis total y no suelen desaparecer hasta 2 - 4 semanas después de haber terminado el tratamiento. Algunos síntomas (como las alteraciones del gusto y olfato y la xerostomía) pueden tardar varios meses en recuperarse ó mantenerse indefinidamente.

La RT de los tumores de cabeza y cuello es la que produce más sintomatología, pudiendo dar de forma aguda mucositis orofaríngea muy dolorosa, disfagia, odinofagia, xerostomía, hipo y disgeusia, y alteración o pérdida de olfato. Los posibles efectos crónicos son: xerostomía (por fibrosis de las glándulas salivares), pérdida del sabor, caries dental, úlceras, osteorradionecrosis mandibular y trismus (por fibrosis muscular).

En la RT abdominal o pélvica (estómago, páncreas, colon, recto, cérvix, útero, próstata) los efectos agudos aparecen en función de la localización de la lesión que se irradie: enteritis, colitis, y proctitis agudas, con diarrea, malabsorción, enteropatía "colerética" por efecto irritativo de las sales biliares no reabsorbidas, alteraciones hidroelectrolíticas, y cistitis.

De forma crónica puede causar también diarrea, malabsorción, enterocolitis, úlceras, estenosis, fístula y cuadros suboclusivos [3]. 


\section{Quimioterapia.}

La aparición de los síntomas y su intensidad dependen de factores como el tipo de fármaco empleado, el esquema de tratamiento (mono o poliquimioterapia), la dosis, la duración y las terapias concomitantes, a la vez que de la susceptibilidad individual de cada paciente, en la que influye el estado clínico y nutricional previo. Los agentes quimioterápicos más tóxicos son los citostáticos, pues al actuar sistémicamente sobre las células de proliferación rápida afectan no solo a las células neoplásicas sino también a células no neoplásicas como las de médula ósea, tracto digestivo, folículo piloso, entre otras.

Los efectos adversos que pueden afectar el estado nutricional del enfermo son numerosos: náuseas, vómitos, anorexia, alteraciones del gusto, mucositis, pudiendo aparecer enteritis severa con edema y ulceraciones de la mucosa, causante de malabsorción y diarrea, que puede llegar a ser explosiva, muy intensa y con sangre. Los efectos secundarios más frecuentes de la quimioterapia son las náuseas y los vómitos, que aparecen en más del $70 \%$ de los pacientes [3].

\section{Requerimientos de Energía y Nutrientes}

De acuerdo a las Guías de la Sociedad Europea de Nutrición para pacientes con cáncer, se recomienda que si el gasto de energía en pacientes oncológicos no es medido específicamente a cada paciente se haga un cálculo de 25 - $30 \mathrm{Kcal} / \mathrm{Kg}$ de peso/día [6].

En relación a este cálculo el nivel de evidencia es "bajo" porque solo unos pocos estudios incluyendo un pequeño número de pacientes han evaluado el gasto total de energía en pacientes con cáncer. Sin embargo la fuerza de esta recomendación se basa en su plausibilidad biológica, que se fundamenta en los beneficios conocidos de una nutrición energéticamente adecuada para mantener un estado nutricional estable [7].

En el paciente oncológico que va a recibir dieta se recomienda, el reparto clásico [55 - 60\% de HC, 30 - 35\% de Lípidos (L) y 15\% de Proteínas (P).

No existe evidencia de que el soporte nutricional incremente el crecimiento tumoral.

\section{Requerimiento de Proteína}

En investigaciones realizadas se ha demostrado que una ingesta mayor de proteínas promueve el anabolismo en pacientes oncológicos. Con un nivel de evidencia moderado se recomienda que la dosis de proteína debe ser de $1,5 \mathrm{~g} / \mathrm{Kg} /$ día [8].

La síntesis de proteínas musculares no disminuye completamente en pacientes con cáncer, algunos estudios sugieren que este proceso no está alterado y responde a la suplementación con aminoácidos [9]. En revisiones recientes la dosis de proteína que ayude a alcanzar un balance proteico positivo en pacientes oncológicos sería aproximadamente $2 \mathrm{~g} / \mathrm{Kg} /$ día, excepto en pacientes con función renal alterada sería de $1 \mathrm{~g} / \mathrm{Kg} /$ día. 
Estudios que mencionen la calidad de la proteína en pacientes con cáncer son escasos. De un estudio prospectivo, randomizado y cruzado que involucraba pacientes con adenocarcinoma intraabdominal avanzado, Tayek y Hunter concluyen que nutrición parenteral enriquecida con aminoácidos de cadena ramificada resultaron en incremento de proteínas y de la síntesis de albúmina cuando se comparó a soluciones de aminoácidos estándar [10-12].

\section{Requerimiento de Lípidos y Carbohidratos}

La proporción óptima de carbohidratos y grasas en la alimentación del paciente con cáncer no han sido determinados pero pueden derivarse de argumentos fisiopatológicos. En pacientes con resistencia a la insulina, la captación y oxidación de la glucosa por las células musculares está deteriorada; sin embargo, la utilización de la grasa está normal o aumentada $[13,14]$, lo que sugiere un beneficio para una mayor proporción de grasa en relación a los carbohidratos.

La mayoría de las recomendaciones dietéticas en pacientes con anorexia por cáncer, se centran en incrementar la densidad energética de la dieta y los productos comerciales disponibles son promocionados y elegidos debido a su alta densidad de energía. Es conocido que la disminución del apetito, la saciedad temprana, y la motilidad intestinal reducida, conspiran para limitar la ingesta de alimentos de baja densidad energética.

En 1971, Waterhouse y Kemperman demostraron que la grasa se movilizó de manera eficiente y se utilizó como fuente de combustible en pacientes con cáncer [15]. Del mismo modo, varios autores más tarde demostraron una muy eficiente movilización y oxidación de la grasa endógena en el estado postabsortivo que varía entre 0.7 y $1.9 \mathrm{~g} / \mathrm{kg} /$ día.

Las emulsiones grasas aportan ácidos grasos esenciales. El uso de grandes cantidades de emulsión lipídica a base de soja estándar, sin embargo, ha sido cuestionado debido al alto contenido de PUFA- $\mathrm{N}-6$, que se ha asociado con un aumento en la producción de eicosanoides proinflamatorios [4]. Las emulsiones a base de aceite de oliva contienen aproximadamente $20 \%$ de PUFA N-6 (es decir, suficiente para suministrar los ácidos grasos esenciales) y $65 \%$ de ácido oleico.

Las fórmulas lipídicas que contiene omega 3 , disminuyen la actividad inflamatoria y se recomienda utilizar de 1.5 a 2 gramos diarios en pacientes oncológicos.

Existen ventajas adicionales al reemplazar la glucosa con lípidos en regímenes de nutrición parenteral. Es prudente tratar de limitar los riesgos infecciosos asociados con la hiperglucemia, que, aunque se ha informado principalmente en el contexto no oncológico, puede ser similar en pacientes con cáncer con resistencia a la insulina.

Además, la administración de glucosa tiende a causar un balance de agua positivo. Gamble [16] demostró por primera vez que la glucosa reduce la excreción renal de sodio y, por las mismas razones, la pérdida del fluido extracelular. 


\section{Requerimiento de Mlcronutrientes}

Una premisa general de la práctica de nutrición es proporcionar a todos los pacientes una dieta nutricionalmente adecuada, que incluya todas las clases de Micronutrientes [17], especialmente aquellos que son esenciales en el ser humano. En todas las formas de desnutrición existe un riesgo de déficit de micronutrientes, especialmente de vitaminas solubles en agua.

En cuanto a los requerimientos de los pacientes con cáncer para vitaminas y oligoelementos, confiamos en la revisión de Strohle et al.[18] y declaraciones recientemente informadas por la American Cancer Society "1) En vista del patrón dietético restringido de pacientes con tumores, el uso de un suplemento multivitamínico multimineral en dosis fisiológicas, es decir, cantidades de nutrientes aproximadamente igual que la cantidad diaria recomendada, es de utilidad y de uso seguro. Esto también se aplica a los pacientes con cáncer durante la quimioterapia y la radioterapia".

Para alimentación oral y enteral, los requerimientos diarios de micronutrientes, puede tomarse como referencia las recomendaciones de la OMS / FAO [19] así como sociedades nacionales e internacionales de nutrición.

Del mismo modo, las vitaminas y oligoelementos deben ser generalmente administrados en nutrición parenteral a menos que haya contraindicaciones.

La suplementación de vitaminas y oligoelementos es obligatoria después de una nutrición parenteral de más de 1 semana. Una dosis estándar de vitaminas y oligoelementos basada en las ingestas dietéticas actuales de referencia para la alimentación oral es generalmente recomendada, a menos que ciertas situaciones clínicas requieran otras ingestas. En general, la nutrición parenteral debe considerar también la administración de elementos traza.

Además, con bastante frecuencia se observa deficiencia de vitamina $D$ en pacientes con cáncer, esto se ha asociado con la incidencia y pronóstico del mismo [20].

\section{Conclusiones}

Es necesario establecer una política intrahospitalaria para el diagnóstico temprano y oportuno de la desnutrición en los pacientes oncológicos, tratando de identificar las posibles causas modificables de la desnutrición asociada al cáncer. No hay evidencia que el apoyo nutricional aumente el tamaño tumoral por lo que el cálculo proteico y calórico debe plantearse en propósitos de recuperación nutricional.

\section{Agradecimientos}


Reconocemos a las personas que ayudaron a la compilación bibliográfica de los artículos en la presente revisión, en especial al personal de la biblioteca del Instituto Oncológico Nacional "Dr. Juan Tanca Marengo", Solca-Guayaquil- Ecuador.

\section{Información adicional}

Nota del Editor

La Revista Oncología Ecu

permanece neutral con

respecto a los reclamos

jurisdiccionales en mapas

publicados y afiliaciones

institucionales.
Abreviaturas

IL: Interleucinas

LPL: Lipoproteína Lipasa

TAB: Tejido Adiposo Blanco

TAG: Triacilgliceroles

TNF: Factor de necrosis Tisular

IL: Interleucinas

\section{Archivos Adicionales}

Ninguno declarado por los autores.

\section{Fondos}

Los fondos de la investigación fueron propios de los autores del presente artículo.

\section{Disponibilidad de datos y materiales}

Existe la disponibilidad de los artículos consultados en la presente revisión.

\section{Contribuciones de los autores}

Todos los autores participaron por igual en la concepción de la idea de revisión, recolección de artículos, escritura del artículo. Todos los autores leyeron y aprobaron la versión final del artículo.

\section{Información de los autores}

Dolores Rodríguez Veintimilla, Doctara Jefe del Servicio de Nutrición Clínica y Dietética del Instituto Oncológico Nacional. SOLCA- matriz Guayaquil

E-mail: dra.rodriguezv@yahoo.com (D) https://orcid.org/0000-0001-7030-0461 
Evelyn Frías-Toral, Máster en Investigación Clínica y Epidemiológica, Diplomado Nutrición Enteral y Parenteral. Coordinadora del Comité de Investigación del Instituto Oncológico Nacional. SOLCA- matriz Guayaquil

E-mail: evelynft@gmail.com

Sergio Santana Portobén, Médico del Hospital Pediátrico Docente Juan Manuel Marquez de La Habana. Editor-Ejecutivo de la Revista Cubana de Alimentación y Nutrición. Cuba.

E-mail: ssergito@infomed.sld.cu.

Aprobación de ética y consentimiento para participar

No aplica a un artículo de revisión.

\section{Consentimiento para publicación}

No aplica.

\section{Referencias}

1. Gómez C, Rodríguez L, Luengo L, Zamora P, Celaya S, Zarazaga A. et al. Intervención nutricional en el paciente oncológico adulto. Barcelona: Glosa; 2003.

2. Freijer K, Tan S, Koopmanschap M, Meijers J, Halfens R, Nuijten M. The economic costs of disease related malnutrition. Clin Nutr. 2013; 32(1): 136-41.

3. García P, Parejo J, Pereira J. Causas e impacto clínico de la desnutrición y caquexia en el paciente oncológico. Nutr Hosp. 2006; 21 (S3): 10-16.

4. Farhangfar A, Makarewicz M, Ghosh S, Jha N, Scrimger R, Gramlich L, et al. Nutrition impact symptoms in a population cohort of head and neck cancer patients: Multivariate regression analysis of symptoms on oral intake, weight loss and survival. Oral Oncol. 2014; 50 (9): 877-883.

5. Bozzeti F, Arends J, Lundholm K, et al. ESPEN Guidelines on Parenteral Nutrition: Non-surgical oncology. Clin Nutr. 2009; 28: 445-54.

6. $\quad$ Argilés J. Fisiopatología de la Caquexia cancerosa. Nutr Hosp. 2006; 21(Supl. 3): 4-9.

7. Guyatt G, Oxman A, Schünemann H, Tugwell P, Knottnerus A. GRADE guidelines: a new series of articles in the Journal of Clinical Epidemiology. J Clin Epidemiol. 2011; 64 :380-2.

8. Arends J, Bachmann P, Baracos V, Barthelemy N, Bertz H, Bozzeti F, et al. ESPEN Guidelines on Nutrition in cancer patients. Clin Nutr. 2017; 36 (1): 11-48.

9. Marín $\mathrm{M}$, et al. Evaluación del riesgo nutricional e instauración de soporte nutricional en pacientes oncológicos, según el protocolo del grupo español de Nutrición y Cáncer. Nutr Hosp. 2008; 23(5): 458-468. 
10. Bozzetti $\mathrm{F}$, Bozzetti $\mathrm{V}$. Is the intravenous administration of amino acid adequate in cancer patients? A critical appraisal of literature. Clin Nutr. 2013; 32:142-6

11. Tayek J, Bistrian B, Hehir D, Martin R, Moldawer L, Blackburn G. Improved protein kinetics and albumin synthesis by branched chain aminoacid-enriched total parenteral nutrition in cancer cachexia. A prospective randomized crossover trial. Cancer. 1986; 58: 147-57.

12. Hunter D, Weintraub M, Blackburn G, Bistrian B. Branched chain amino acids as the protein component of parenteral nutrition in cancer cachexia. Br J Surg. 1989;76:149-53.

13. Cáceres $\mathrm{H}$, Neninger $\mathrm{E}$, Menéndez $\mathrm{Y}$, Barreto J. Intervención Nutricional en el paciente con cáncer. Rev cubana med. 2016; 55 (1): 59-73.

14. Arcidiacono B, liritano S, Nocera A, Possidente K, Nevolo M, Ventura V, et al. Insulin resistance and cancer risk: an overview of the pathogenetic mechanisms. Exp Diabetes Res. 2012; 2012: 789174. doi: $10.1155 / 2012 / 789174$

15. Waterhouse C, Kemperman J. Carbohydrate metabolism in subjects with cancer. Cancer Res. 1971; 31 : $1273-8$.

16. Gamble J. The Harvey Lectures, Series XLIII, 1946-1947: physiological information gained from studies on the life raft ration. Nutr Rev. 1989; 47: 199-201.

17. Vanek V, Borum P, Buchman A, Fessler T, Howard L, Shenkin A, et al. Novel Nutrient Task Force, Parenteral Multi-Vitamin and Multi-Trace Element Working Group and American Society for Parenteral and Enteral Nutrition (A.S.P.E.N.) Board of Directors. A.S.P.E.N. position paper: recommendations for changes in commercially available parenteral multivitamin and multi-trace element products. Nutr Clin Pract. 2012;27: 440-91.

18. Strohle A, Zanker K, Hahn A. Nutrition in oncology: The case of micronutrients (Review). Oncol Rep. 2010; 24: 815-28.

19. WHO. Diet, nutrition and the prevention of chronic diseases. Report of a Joint FAO/ WHO Expert consultation. WHO Technical report series 916. WHO: Geneva, 2003.

20. Arends J. Vitamin D in oncology. Forsch Komplementmed. 2011; 18: 176-184. 